evacuation drills, especially the clearance of an intensive care or an operating room; access to evacuation routes; visibility of safety guidelines; need of specific evacuation equipment for the movement of patients; mission and tasks of the hospital's first response team and the medical incident manager; communication and information flow and the establishment of the hospital's coordination committee.

Conclusion: 1. Simulated hospital evacuation exercises increased the hospital emergency preparedness, awareness and response to disasters within the hospital, in particular in a CCD, otherwise difficult to assess. 2. All three CCD experienced the same challenges and identified similar flaws. 3. A hospital disaster exercise manual might be of valuable help. Prehosp Disaster Med 2011;26(Suppl. 1):s40-s41 doi:10.1017/S1049023X11001439

(A143) European Project SICMA (Simulation of Crisis Management Activities) for Medical Management of Maxi Emergency Trauma Patients S. Magalini, ${ }^{1}$ M. Di Mugno, ${ }^{1}$ A. De Gaetano, ${ }^{2}$

G. La Posta, ${ }^{2}$ D. Sermoneta, ${ }^{1}$ D. Gui ${ }^{1}$

1. Surgery, Rome, Italy

2. Rome, Italy

Introduction: Modern emergencies and disasters are progressively changing from relatively simple, predictable events controllable with standard management solutions to complex critical situations for which managers and first responders require innovative and affordable tools.

Methods: The European Project SICMA (Simulation of Crisis Management Activities) provides a modeling of the behavior of the entire Health Service System during field emergency operations, as well as the rules it operates by. Use of simulation technologies offers a significant improvement on current management activities allowing decision makers to confront several organizational alternatives not only with static situations but with evolving scenarios.

Results: SICMA simulates main structural and behavioral elements of maxi emergency and mass casualties, from individual casualties and evolution of their health status, activity of the police force and fire brigade on the accident site, crowd dynamics, sanitary personnel expertise, to ambulance and helicopter transportation depending on traffic and weather conditions. The system also simulates rescue doctrines (i.e. "Casualty Clearing Station" or "Scoop and run"), transportation priorities according to color codes, doctrines for assignment of new casualty to neighboring hospitals, hospital resources and involvement, final clinical outcome of individual casualties. Patient health status and physiological reserve of single casualty is based on the ABCD ATLS system, considering with a simple algorithm both level of damage and rate of worsening in time.

Conclusion: Utilizing this simulation system, managers who predispose organizational and logistic procedures may modify the main elements in order to identify the optimal resource allocation and the best procedures to save the most human lives.

Prehosp Disaster Med 2011;26(Suppl. 1):s41

doi:10.1017/S1049023X11001440
(A144) Health Status Casualty Model for Simulation of Crisis Management Activities (EU SICMA Project)

M. Di Mugno, ${ }^{1}$ S. Magalini, ${ }^{1}$ A. De Gaetano, ${ }^{2}$

G. La Posta ${ }^{2}$ D. Sermoneta, ${ }^{1}$ D. Gui ${ }^{1}$

1. Surgery, Rome, Italy

2. Rome, Italy

Introduction: The European Project SICMA (Simulation of Crisis Management Activities) provides a modeling of the behavior of the entire Health Service System during field emergency operations, as well as the rules it operates by. The first step toward chain procedure modelling in the management of major emergencies is the representation of a traumatized patient whose health status can be followed in time during simulation. Since management of the trauma patient follows criteria of stabilization of main physiological functions, a trauma patient model was developed based on fundamental pathophysiological functions independently of specific lesion characterization. Methods: Each patient's health status was modelled according to 5 parameters (ATLS): $\mathrm{A}$ (airway), $\mathrm{B}$ (breathing), $\mathrm{C}$ (circulatory), $\mathrm{D}$ (disability), E(Exposure). Patient samples are extracted from a 10.000 .000 patient database, generated by considering real anatomical lesions compatible with type and severity of considered scenarios (explosion, building collapse, fire, gunfight). Simulated lesion characteristics were then converted to pathophysiological parameters. Each physiological compensation parameter was represented by: (1) baseline value expressed as percentage of altered function; (2) function reduction rate over time, obtained by a mathematical approximation of clinical worsening. From level of function, rate of worsening and function-specific death thresholds, estimated time-to-death according to sustained damage is computed.

Results: This model allows simulation of evolution of patient health status both in absence of medical care, but also under therapy, in terms of immediate increment of each single parameter ("temporary" treatment), and of reduction or zeroing of parameter dec14rement rate ("definitive" treatment).

Conclusion: This model, based on evaluation of physiological parameters, presents an advantage over the consideration of single lesions, because simulating logical procedures that guide treatment choice in real situations can provide a more accurate assessment of casualities for those actors assigned to management of major emergencies.

Prehosp Disaster Med 2011;26(Suppl. 1):s41

doi:10.1017/S1049023X11001452

(A145) Simulation for the Assessment and Optimization of Medical Disaster Management

E.L. Dhondt, ${ }^{1}$ F. Van utterbeek, ${ }^{2}$ C. Ullrich, ${ }^{2}$

M. Debacker ${ }^{3}$

1. Emergency and Disaster Medicine, Brussels, Belgium

2. Brussels, Belgium

3. Military Hospital, Brussels, Belgium

Background: The ultimate goal of medical disaster management must be to predictably orchestrate transition from "standard of care" to "sufficiency of care" using evidence-based methods. However, neither descriptive reports of disaster responses nor epidemiological studies investigating disaster risk factors have been able to provide validated outcome measures as to what constitutes a "good" disaster response. Moreover, it either has 
been considered impossible, ethically inappropriate, or both, to identify experimental and control groups essential for hypothesis testing for the conduct of scientific randomized controlled clinical trials.

Objective: The aim of this study was to identify a number of performance and outcome indicators and define optimal disaster response and management decision-making for various disaster scenarios using simulation optimization.

Methods and Results: A system model of medical disaster management was designed, and victim models and performance and outcome indicators were developed. Various mass-casualty and large-scale disaster scenarios were developed, including: (1) a hospital emergency incident/disaster; (2) a CBRNE incident; (3) an airplane crash and airport disaster; (4) a mass gathering; and (5) a military battlefield mass casualty. Using "Discrete Event Driven Simulation", multiple replications were made for different decision-making modalities, different resource allocations, and different disaster response procedures. Statistical analysis and optimization techniques were applied to achieve the best available setting of parameters of the simulation model. In such a way, the "Medical Disaster Management Simulator" runs the "missing experimental studies" in a simplified artificial simulated disaster environment.

Conclusions: Simulation optimization is an adequate tool for judging and evaluating the effectiveness and adequacy of health and relief services provided during disaster medical response. Evidence-based recommendations and codes of best practice were formulated for optimal medical disaster and military battlefield management in different large-scale event scenarios as well as for teaching, training, and research in medical disaster management. Prehosp Disaster Med 2011;26(Suppl. 1):s41-s42 doi:10.1017/S1049023X11001464

(A146) Disaster Patient Tracking-Local, State and Federal Interoperability during a Multi-Hospital Evacuation Exercise

K. Andress, ${ }^{1}$ E. Downey ${ }^{2}$

1. Department of Emergency Medicine, Shreveport, United States of America

2. Department of Environmental Sciences, New Orleans, United States of America

Introduction: Associated with hospital evacuation is the need to track multiple patient evacuees from point of origination to final hospital reception. Patient tracking, a component of the hospital emergency operations plan, is vital to patient care; family association, resource management, financial reimbursement, risk management, and repatriation. Tracking strategies and plans can include a variety of vendors, hardware, software, and coordination issues. Hospital evacuee tracking plans and platforms exist at multiple jurisdictional levels but may not be interoperable.

Methods: Three patient tracking platforms representing a local, state and federal application were used during a multi-hospital evacuation exercise, initiated in New Orleans, Louisiana, May 2010. Simulated patients were flown and tracked to multiple patient reception centers in the southern United States, including the Federal Coordinating Center in Shreveport, Louisiana, and receiving National Disaster Medical System hospitals. This review summarizes tracking operations, patient data characteristics captured and interoperability at the Shreveport reception location.

Results: 7 New Orleans hospitals entered 51 patients for evacuation into Louisiana's web-based, At-Risk-Registry (ARR) database including 8 patient identifiers each. ARR data was shared with federal and Louisiana Region 7 patient evacuee receivers for flight manifest construction and reception planning. 34 ARR evacuee patients were indicated for the Shreveport, Louisiana, reception site. 34 patients with 6 identifying characteristics were entered from ARR into EMTrack, the local patient tracking system. A C130 arrived with a TRAC2ES manifest of 20 simulated patients with 6 patient data characteristics. The local tracking system was reconfigured for the hardcopy manifest; simulated patients were received at the airport; transported and received at local hospitals.

Conclusions: Tracking system interoperability may be challenged by tracking technologies, jurisdictional requirements and degree of implementation at the local, state and federal level. Tracking should be standardized based on national recommendations with local systems remaining flexible for just-in-time requirements.

Prehosp Disaster Med 2011;26(Suppl. 1):s42 doi:10.1017/S1049023X11001476

\section{(A147) Pediatric Medical Surge: An Exercise Evaluation Guide} E.K. Weber

Bureau of Public Health Preparedness \& Emergency Response, Chicago, United States of America

A guide was created by the Chicago Healthcare System Coalition for Preparedness and Response to help hospitals and health facilities design, implement, and evaluate emergency exercises following the US Homeland Security Exercise and Evaluation Program (HSEEP) format. The HSEEP provides a standardized policy, methodology, and terminology for exercise design, development, conduct, evaluation, and improvement planning. As a part of a toolkit for hospital use, the pediatric at-risk population is represented with an Exercise Evaluation Guide titled "Pediatric Medical Surge". Pediatric Medical Surge is defined as the rapid expansion of the capacity of the existing healthcare system in response to an event that results in an influx of children and an increased need for personnel (clinical and non-clinical), support functions (laboratory and radiological), physical space (beds, alternate care facilities), and logistical support (clinical and non-clinical equipment and supplies). The Exercise Evaluation Guide is fully customizable and includes the following activity sections: (1) Pediatric Pre-Event Mitigation and Preparedness; (2) Incident Command; (3) Pediatric Bed Surge Capacity; (4) Pediatric Surge Staffing Procedure; (5) Pediatric Decontamination; (6) Receive, Evaluate, and Treat Pediatric Surge Casualties; (7) Provide Pediatric Surge Capacity for Behavioral Health Issues; and (8) Demobilization. Each of these sections includes a number of exercise tasks and details the potential tasks/observation keys that are completely modifiable in an electronic format. All or a limited number of these activity sections can be used in an exercise. Following the Activity and Tasks, a section for Observations is provided, and 